\title{
Christèle Couleau-Maixent, Balzac. Le roman de l'autorité. Un discours auctorial entre sérieux et ironie
}

Aude Déruelle

\section{(2) OpenEdition}

1 Journals

\section{Édition électronique}

URL : http://journals.openedition.org/studifrancesi/7625

DOI : 10.4000/studifrancesi.7625

ISSN : 2427-5856

Éditeur

Rosenberg \& Sellier

\section{Édition imprimée}

Date de publication : 1 décembre 2009

Pagination : 646-647

ISSN : 0039-2944

\section{Référence électronique}

Aude Déruelle, «Christèle Couleau-Maixent, Balzac. Le roman de l'autorité. Un discours auctorial entre sérieux et ironie », Studi Francesi [En ligne], 159 (LIII | III) | 2009, mis en ligne le 30 novembre 2015, consulté le 09 janvier 2021. URL : http://journals.openedition.org/studifrancesi/7625 ; DOI : https:// doi.org/10.4000/studifrancesi.7625

Ce document a été généré automatiquement le 9 janvier 2021.

\section{(c)}

Studi Francesi è distribuita con Licenza Creative Commons Attribuzione - Non commerciale - Non opere derivate 4.0 Internazionale. 


\title{
Christèle Couleau-Maixent, Balzac. Le roman de l'autorité. Un discours auctorial entre sérieux et ironie
}

\author{
Aude Déruelle
}

\section{RÉFÉRENCE}

CHRISTÈLE COULEAU-MAIXENT, Balzac. Le roman de l'autorité. Un discours auctorial entre sérieux et ironie, Paris, Honoré Champion, 2007, pp. 855.

1 À tous égards, l'ouvrage de Christèle Couleau-Maixent constitue une somme. Par l'ampleur du volume certes, mais également par la qualité des analyses, par la multiplicité des phénomènes abordés, par la manière enfin et surtout dont l'auteur rassemble un certain nombre d'avancées de la critique balzacienne de ces dernières années pour en proposer à la fois une synthèse et une réinterprétation globale. Depuis quinze ans en effet ont fleuri des études (articles ou thèses) de la poétique balzacienne, qui ont renouvelé l'approche de cet auteur. L'un des mérites de C. Couleau-Maixent est de se nourrir de toutes ces analyses, en se donnant pour objet un phénomène qui invite à une approche générale de l'ensemble des procédés poétiques: le discours auctorial. Celui-ci ne se réduirait pas au discours du narrateur, mais engloberait les propos des personnages, dès lors qu'ils manifestent une autorité, un «exercice de la fonction idéologique» (p. 44), telle qu'a pu la définir Gérard Genette dans Figures III. Le principal objet de l'ouvrage est d'ailleurs d'explorer le caractère «protéiforme» (p.126), «composite» (p. 132), du discours auctorial balzacien.

2 L'ouvrage se compose de trois parties. La première vise tout d'abord à cerner la notion même de «discours auctorial», en la confrontant à d'autres expressions, souvent utilisées par la critique balzacienne (doxa, notamment). S'appuyant d'intelligente façon sur les propos de Foucault, C. Couleau montre que le discours auctorial n'est pas l'expression d'une autorité par ailleurs constituée, mais le lieu où s'articule, se compose 
(et se décompose parfois) une posture auctoriale. On s'attardera notamment sur le tableau de la page 75 qui délimite astucieusement le champ idéologique de ce discours auctorial, entre savoir et axiologie. Une fois la notion définie, C. Couleau-Maixent la fait interagir avec le genre romanesque, tel qu'a contribué à l'instituer Balzac, en s'interrogeant sur le choix du narratif, et non du discursif pur (on pense à ce sujet à l'analyse désormais classique de Jacques Neefs, Les trois étages du mimétique dans "La Comédie humaine", in Balzac. CEuvres complètes. Le "Moment» de "La Comédie humaine", éd. Cl. Duchet et I. Tournier, Saint-Denis, P.U.V., 1993, pp.149-156.). Et de traquer les différentes formes du discours auctorial (intrusions d'auteur, commentaires des personnages), ses lieux d'apparition (incipit, explicit, glose), etc... L'analyse d'un extrait d'Une Fille d'Ève (p. 274 sq.) vient illustrer de façon remarquable ces différentes configurations du discours auctorial, en une attention au texte qui rappelle l'étude du discours balzacien menée par Éric Bordas (Balzac, discours et détours. Pour une stylistique de l'énonciation romanesque, P.U.M., 1997).

3 La deuxième partie, consacrée à «la fabrique de l'autorité», s'attarde d'abord sur les «visages de l'autorité» construits par le texte: les indices, les marques du pouvoir tels qu'ils s'incarnent dans les personnages. L'analyse peut parfois paraître quelque peu déplacée par rapport à l'objet d'étude même, et d'ailleurs, concède C. Couleau-Maixent, «les représentants du pouvoir ne sont pas les porte-parole privilégiés de La Comédie humaine» (p. 300). C'est plutôt du côté des représentants du savoir (médecin, prêtre, juriste) ou de certains marginaux (le forçat) que se constitue le pôle d'autorité, même si «le tout n'est pas de savoir: encore faut-il faire savoir que l'on sait» (p. 335). Cette étude aboutit à des schémas (p. 371) qui dressent la cartographie de l'autorité du personnage selon ses traits de caractère et ses capacités et qui évoquent l'analyse du personnage et de ses compétences par Philippe Hamon (Texte et Idéologie, P.U.F., 1984, ch.3). Enfin, l'ouvrage s'attarde sur le cas particulier des "personnages génériques» (l'observateur, le poète, le voyageur), selon la formule de $\mathrm{C}$. Couleau-Maixent à présent régulièrement utilisée par les études balzaciennes, même s'il convient peut-être de mieux distinguer le personnage générique du «type», l'expression «personnage générique typique» (p. 405), à propos d'un ouvrier, étant quelque peu déroutante. Le «personnage générique» est un adjuvant du discours auctorial, le «type» est un personnage qui a pour fonction de représenter une catégorie d'individus plus ou moins semblables, et dont les traits sont suffisamment proches pour légitimer une telle assimilation. La fin de la deuxième partie s'attache à étudier les différents procédés du discours auctorial, dans une perspective somme toute rhétorique: cette étude a plus les vertus de la synthèse que de la nouveauté, et la référence très présente à l'ouvrage de Susan Suleiman (Le Roman à thèse ou l'autorité fictive, P.U.F., 1983) n'est peut-être pas des plus pertinentes pour aborder le texte balzacien.

4 La dernière partie saisit le discours auctorial comme discours «à l'articulation du roman et du monde» (p.587) et étudie la manière dont le genre romanesque pose les questions du savoir (l'encyclopédie) et de la pédagogie (la prise en compte du lecteur): qu'est-ce que le roman a à nous dire sur le monde, sur le réel? C. Couleau-Maixent dresse ici une brève cartographie des connaissances prises en charge par le roman balzacien: sociologie des envers du monde, des petits riens qui font tout, vision historique du passé et du présent, géographie du monde et de ses discours. Mais le roman ne saurait prendre en charge tout le réel, tout le XIX siècle: malgré son ambition totalisante, il est nécessairement frappé d'incomplétude. De là des stratégies visant à pallier ce défaut inévitable: dire que l'on ne peut tout dire, comprimer le réel en type et 
en cas, édicter des lois, et... La Comédie humaine se charge ainsi de «modéliser» le réel selon ses configurations possibles: la démonstration de C.Couleau-Maixent sur la question du mariage et de l'adultère (p. 712) est à cet égard remarquablement bien menée. Le discours auctorial permet de «plier» le réel (p.724), le rendant plus accessible et plus lisible. On peut juste regretter que toute cette partie ne fasse pas référence aux travaux, d'inspiration très proche, de Boris Lyon-Caen (Balzac et la comédie des signes. Essai sur une expérience de pensée, Saint-Denis, P.U.V., 2006). L'ouvrage s'achève sur l'étude du roman balzacien comme tribune idéologique, en revenant sur la question de l'ironie.

5 Le livre de C. Couleau-Maixent, on l'aura compris, a le double mérite de nous donner une vue d'ensemble de la poétique balzacienne, tout en proposant une série d'analyses précises de qualité. Face à un ouvrage d'une telle ampleur, et dont l'ambition est légitime, tout au plus peut-on émettre quelques réserves de détail. L'analyse de la focalisation demanderait à être reprise, quelques erreurs s'étant glissées dans l'usage de la notion de «focalisation externe», il est vrai de maniement un peu délicat (p. 164). On peut s'interroger aussi sur la pétition de principe de C. Couleau-Maixent, qui déclare au seuil de son livre que le discours auctorial englobe discours du narrateur et propos des personnages - du moment que ceux-ci manifestent une autorité - mais qui ne cesse, au cours de son analyse, de montrer a contrario que le discours du personnage «est une version dégradée du discours auctorial» (p.154): «voir le monde par les yeux d'un personnage n'est sans doute pas la meilleure façon d'accéder à un savoir objectif: sa subjectivité conditionne le commentaire auquel il se livre, sa compétence remet en doute la validité, ses motivations propres en altèrent l'intention» (p. 168). Et de dire encore: «le rôle du narrateur est essentiel, puisqu'en dernier recours c'est lui qui tranche» (p. 374) (la légitimation par le narrateur est essentielle. Ce qui revient à dire ce que nous énoncions dans notre livre, «la parole des personnages est entachée de non-légitimité», Balzac et la digression. Une nouvelle prose romanesque, Saint-Cyr-sur-Loire, Christian Pirot, 2004, p. 146, formulation pourtant critiquée par C. Couleau-Maixent). Enfin, on pourra regretter l'absence d'une chronologie interne du discours auctorial balzacien: la posture auctoriale développée dans La Peau de chagrin (1831) n'est sans doute pas assimilable à celle qui figure dans La Cousine Bette (1846), ni à celle élaborée dans Le Père Goriot (1835) ou Illusions perdues (1837-1843). Il en va de même des places respectives du sérieux et de l'ironie, et surtout de la manière dont ces deux modalités du discours interagissent: la saisie d'une évolution au sein de la création balzacienne aurait été intéressante. Ces quelques réserves de détail ne sauraient toutefois occulter l'incontestable réussite de l'ensemble. 